\title{
On the brightness and velocity structure of solar granulation
}

\author{
J. Hirzberger ${ }^{\star}$ \\ Institut für Geophysik, Astrophysik und Meteorologie, Universitätsplatz 5, 8010 Graz, Austria
}

Received 22 April 2002 / Accepted 14 June 2002

\begin{abstract}
A 45 min time series of two-dimensional spectra has been obtained with the Vacuum Tower Telescope at the Observatorio del Teide in Izaña, Tenerife. Scans over the non-magnetic Fe I 5576 Å line of a quiet granular field at disk center were taken simultaneously with a time series of broad band images. From the spectra intensity and velocity maps have been calculated at different line-depths. From the white light images granular shapes have been computed by means of an automatic image segmentation algorithm. A statistical analysis of the intensity and velocity distribution in the detected granular shapes has been carried out. Intensities and velocities are well correlated at low photospheric levels. In the higher photosphere the intensity pattern dissolves whereas the velocities show almost no variation within the probed height interval. The intensity excess of small granules dissolves at lower heights than that of larger ones. The intensity and velocity distribution within the granules depends on the granular size. In smaller structures the maximum intensities and velocities are located close to the granular barycenters whereas for larger granules the maxima are shifted towards the granular boundaries. The width of the transition zone between granules and intergranular lanes is independent on the granular intensity and is constant at approximately $0{ }^{\prime} 5$. The time evolution of the granular pattern shows a clear dependence of the lifetime of structures on the spatial wavenumber. The $e$-folding times of the temporal coherences decrease according to a power law with an exponent of $\beta=3 / 2$ which is incompatible with the Kolmogorov energy spectrum of homogeneous and isotropic turbulence and might be taken as a hint against the overall turbulent character of granular motions.
\end{abstract}

Key words. Sun: photosphere - Sun: granulation - techniques: spectroscopic - techniques: image processing

\section{Introduction}

The cellular pattern of the quiet solar photosphere can be explained by an overshoot of convective motions into a stable atmosphere. This implies that the structure of solar granulation should be determined by the conditions in the convection zone beneath the photosphere, i.e., assuming Bénard-like convection, that the cell sizes should be comparable to the depth where the hydrogen ionization region is located (Simon \& Leighton 1964). Contradictory to this view, numerical simulations of solar granulation (Stein \& Nordlund 1989, 1998) show that the granular pattern is mainly a surface phenomenon driven by effective radiative cooling in the photosphere. The decrease of pressure in a cooled fluid parcel leads to a horizontal displacement away from warmer regions which triggers the spontaneous formation of localized downflowing plumes due to Rayleigh-Taylor instabilities. The granules (upflow regions) are, therefore, only a secondary phenomenon balancing the radiative loss in the photosphere and ensuring mass conservation in the convective layer (Rast 1995, 1999b). Observational studies of e.g. Rimmele et al. (1995) and Strous et al. (2000) show that the spontaneous formation of downflowing plumes does not only play a dominant role in the development of the convective motions close to the solar surface, but they are also

\footnotetext{
* e-mail: jkh@igam.uni-graz.at
}

expected to be efficient sources of acoustic waves on the Sun. This result is also supported by numerical models of e.g. Rast (1999a) or Stein \& Nordlund (2001).

Since the Reynolds number in the lower solar photosphere is approximately $10^{12}$, the granular flow field is expected to be highly turbulent. This is supported by the analysis of power spectra derived from data obtained with the Multichannel Subtractive Double Pass (MSDP) spectrometer by Espagnet et al. (1993) and from white light images obtained at the Swedish Vacuum Solar Telescope by Hirzberger et al. (1997). Opposite to this view, results from numerical granulation models (e.g. Steffen et al. 1989; Stein \& Nordlund 1998; Gadun et al. 2000) show much less turbulence as expected or even almost laminar granular flow fields. Nordlund et al. (1997) interprete this lack of turbulence as produced by the small pressure scale height in the solar photosphere and, thus, by the rapid expansion of upflowing matter when it enters into higher photospheric levels. Vice versa, increased turbulence should be visible in the intergranular lanes. This idea is supported by observations of e.g. Nesis et al. $(1993,1999)$ and by the numerical models of Rast (1995).

Another approach to understand the phenomenon of solar granulation is the analysis of the behaviour of individual granules and the application of statistical methods to the resulting physical quantities such as lifetimes, sizes, and intensities of granules. Statistical studies of granular properties have 
been carried out, among others, by Mehltretter (1978), Dialetis et al. (1986), Roudier \& Muller (1987), Title et al. (1989), and Hirzberger et al. (1997, 1999). In a recent study performed by Berrilli et al. (2002) spectral scans across several absorption lines have been used to obtain the (line-of-sight) flow velocity as an additional parameter for a statistical analysis.

Although the list of observational studies on solar granulation is quite long (for further references see the review articles of Spruit et al. 1990 and Muller 1999) our knowledge about the substructure of solar granules is still incomplete. Even in most of the statistical analyses individual granules are treated as objects with several physical properties but no internal variation of them. An attempt to analyze the intensity inhomogenities within a large granule using isophote contours was made by Bray \& Loughhead (1984). However, the reason for this lack of knowledge is the disadvantageous ratio between granule sizes (about one arcsecond) and spatial resolution of present (and past) solar data (approximately 0.2 in the very best cases).

More about the granular substructure can be stated from coherence analyses as carried out by e.g. Deubner (1988), Nesis et al. (1988), Komm et al. (1990) from slit spectrograms, Espagnet et al. (1995) from MSDP data, and by e.g. Salucci et al. (1994), Hirzberger et al. (2001) from two-dimensional spectrograms obtained with tunable narrow band filters based on Fabry-Perot interferometers. From these studies two main results can be extracted: (i) the velocity field retains its cellular character in the upper photosphere (although larger structures extend into higher levels than smaller ones, see Komm et al. 1991) whereas the intensity pattern vanishes rapidly and becomes even inverted in the uppermost photospherical levels higher than about $250 \mathrm{~km}$; (ii) the coherence between temperature (intensity) fluctuations and vertical velocity fluctuations is high and the phase between them is zero only for structures larger than about 0.8 up to $1^{\prime \prime}$. Most of the above mentioned studies show that the coherence drops for smaller structures which generally is explained by turbulent flows which become dominant at these structural scales. However, Hirzberger et al. (2001) have shown - using the same data as used in the present paper - that the coherence between photospheric intensity and velocity fluctuations remains high for structures with sizes far below one arcsecond, i.e. until the resolution limit of the data which is approximately $0 . .4$.

The aim of the present paper is to carry out a statistical analysis of the intensity and velocity patterns from different photospheric levels and to show some properties of the distribution of intensities and velocities within granular structures.

\section{Observations and data reduction}

The observations were carried out on October 23rd, 1999, with the "Göttingen" Fabry-Perot Interferometer (FPI) in the $70 \mathrm{~cm}$ Vacuum Tower Telescope (VTT) at the Observatorio del Teide, Tenerife. The telescope was pointed to a quiet granular region at disk center. A 45 min time series of spectral scans across the non-magnetic Fe I line at $\lambda=5576 \AA$ together with simultaneously exposed bursts of broad band images ( $100 \AA F W H M)$ centered at the same wavelength have been obtained. The exposure time for both types of images was $20 \mathrm{~ms}$. The time between the starts of two subsequent spectral scans was $70 \mathrm{~s}$. The pixel size is $0{ }^{\prime} 1$ and the field of view of the raw data is 384 pixel $\times 286$ pixel in size. The iron line has been scanned at 11 spectral positions separated by $\Delta \lambda=35.25 \mathrm{~m} \AA$ and the actual $F W H M$ of the FPI was approximately $52 \mathrm{~m} \AA$.

The data have been carefully corrected for dark offset, flat fields and global image motions. In addition, the narrow band images have been corrected for the transmission curve of the pre-filters and for the wavelength shift across the field of view which appears due to the mounting of the FPIs in the collimated, parallel beam of the setup.

The broad band data have been reconstructed for the optical transfer functions of the telescope and the Earth's atmosphere applying speckle interferometric techniques as described in de Boer (1993). Since the narrow band images have been simultaneously exposed with the broad band ones, a quasi-speckle reconstruction as described in Krieg et al. (1999) could be applied to them. For a more detailed description of the observation procedures and the data reconstruction techniques see Hirzberger et al. (2001).

Line profiles at each pixel of the field of view can be retrieved from the narrow band images. Since the resolution of the data is not expected to be better than 0.3 and for improving the signal-to-noise ratio, the line profiles have been averaged by a 3 pixel $\times 3$ pixel boxcar smoothing over the field of view. From each of the resulting profiles the line bisector has been computed providing intensities and line-of-sight velocities at different heights, $i=\left(I-I_{\mathrm{lc}}\right) /\left(I_{\mathrm{c}}-I_{\mathrm{lc}}\right) \cdot 100[\%]$, in the line profiles. (The quantity $I_{\mathrm{lc}}$ denotes the line center intensity, $I_{\mathrm{c}}$ is the intensity of the local continuum, and $I$ is the measured intensity in the line profile.) An absolute velocity reference cannot be determined from the data because only relative wavelengths have been obtained. The zero points of the velocity maps have, therefore, been defined as the average values in the field of view. The precision of the velocity measurements, derived from noise, is in the range $\Delta v_{\text {rms }} \approx \pm 50 \mathrm{~m} \mathrm{~s}^{-1}$. The intensity maps have been normalized to their mean values.

Since the Airy functions - which describe the transmission curves of the FPIs - have extended wings, the response functions corresponding to the intensity maps are relatively broad and so the separation of the photospheric heights where the light is emitted from is rather low. This problem has been overcome by calculating linear combinations of images from different line depths. For the present study intensity maps, $I_{50}$, from $i=50 \%$ (barycenter of the response function at $h_{\mathrm{bc}}=53 \mathrm{~km}$ above the continuum level) and intensity maps $I_{0}=I(i=0 \%)-0.2 \cdot I(i=25 \%)-0.15 \cdot I(i=75 \%)$ with $h_{\mathrm{bc}}=320 \mathrm{~km}$ have been computed. The width of the velocity response functions is much smaller than that of the corresponding intensity response functions. Therefore, a linear combination of several velocity maps is not necessary. For the present study velocity maps, $v_{0}$, from $i=0 \%\left(h_{\mathrm{bc}}=250 \mathrm{~km}\right)$ and $v_{50}$, from $i=50 \%\left(h_{\mathrm{bc}}=130 \mathrm{~km}\right)$ have been used. (A detailed description of the computation of intensity and velocity maps and plots of the corresponding response functions are given in Hirzberger et al. 2001.)

The last step of the data reduction was to co-align all the maps from the time series and to apply a subsonic filter (with a 
cut-off phase velocity of $5 \mathrm{~km} \mathrm{~s}^{-1}$ ) which removes the influence of solar $p$-modes and of residual image distortions with apparent phase velocities larger than $5 \mathrm{~km} \mathrm{~s}^{-1}$. The subsonic filter was applied to the time series of broad band images, $I_{\mathrm{BB}}$, and to the narrow band intensity and velocity maps. Finally, the data consist of 5 cubes, $I_{\mathrm{BB}}(x, y, t), I_{0}(x, y, t), I_{50}(x, y, t), v_{0}(x, y, t)$, and $v_{50}(x, y, t)$ depending on two spatial dimensions, $x$ and $y$ and the time, $t$. After removing the apodized edges each cube contains 36 images with 260 pixel $\times 190$ pixel in size.

\section{Image segmentation}

The identification of granular structures has been carried out as shown in Hirzberger et al. (1997) based on a Fourier filtering method developed by Roudier \& Muller (1987). The constants of the Fourier filter have been tuned such that the resulting granular shapes match intensity inflection points best as calculated by the application of a Laplacian operator. Again as in Hirzberger et al. (1997) the remaining intergranular lanes outside the detected granular areas have been split into two halves, each "belonging" to its neighbouring granule. Hence, a granular cell can be defined as a granule plus its surrounding part of the intergranular lanes, i.e. the total cell area is $A_{\mathrm{c}}=A_{\mathrm{g}}+A_{\mathrm{ig}}$.

Only the broad band images have been segmented in the above mentioned way. An example is shown in Fig. 1. The fractional granular area amounts to $47.7 \%$ of the total area. This value is strongly dependent on the applied image segmentation algorithm which has to be taken into account when interpreting the results. In the literature much smaller values (e.g. 39\% by Roudier \& Muller 1987) but also much larger values (e.g. $60 \%$ by Bovelet $\&$ Wiehr 2001) can be found. Since for the present study flow maps are available, the detected fractional granular area can be compared with the fractional upflow area in the velocity maps. As shown in Sect. 4 below, the numbers exhibit a good agreement although after a one to one comparison between granular and upflow areas some differences can be detected. The reason for these differences might be: (i) intrinsic differences between broad band intensities and flow velocities; (ii) different formation heights of the broad band intensities and the line asymmetries used for calculating the flow maps; (iii) a slightly better spatial resolution of the broad band data compared to the flow maps (see Hirzberger et al. 2001); (iv) residual non-convective velocities which have not been removed by the applied subsonic filter.

For the statistical analysis all granules truncated by the image boundaries and structures with $A_{\mathrm{g}} \leq 9$ pixel have been removed. After that 5509 granules detected in the broad band images are remaining.

\section{Results}

\subsection{Intensity and velocity distribution functions}

Distribution functions of intensities and velocities in the data cubes are shown in Fig. 2. Intensity and velocity histograms of all pixels in the time series are plotted. At first glance the velocities seem to be symmetrically distributed around zero - this is partially caused by the definition of the zero points
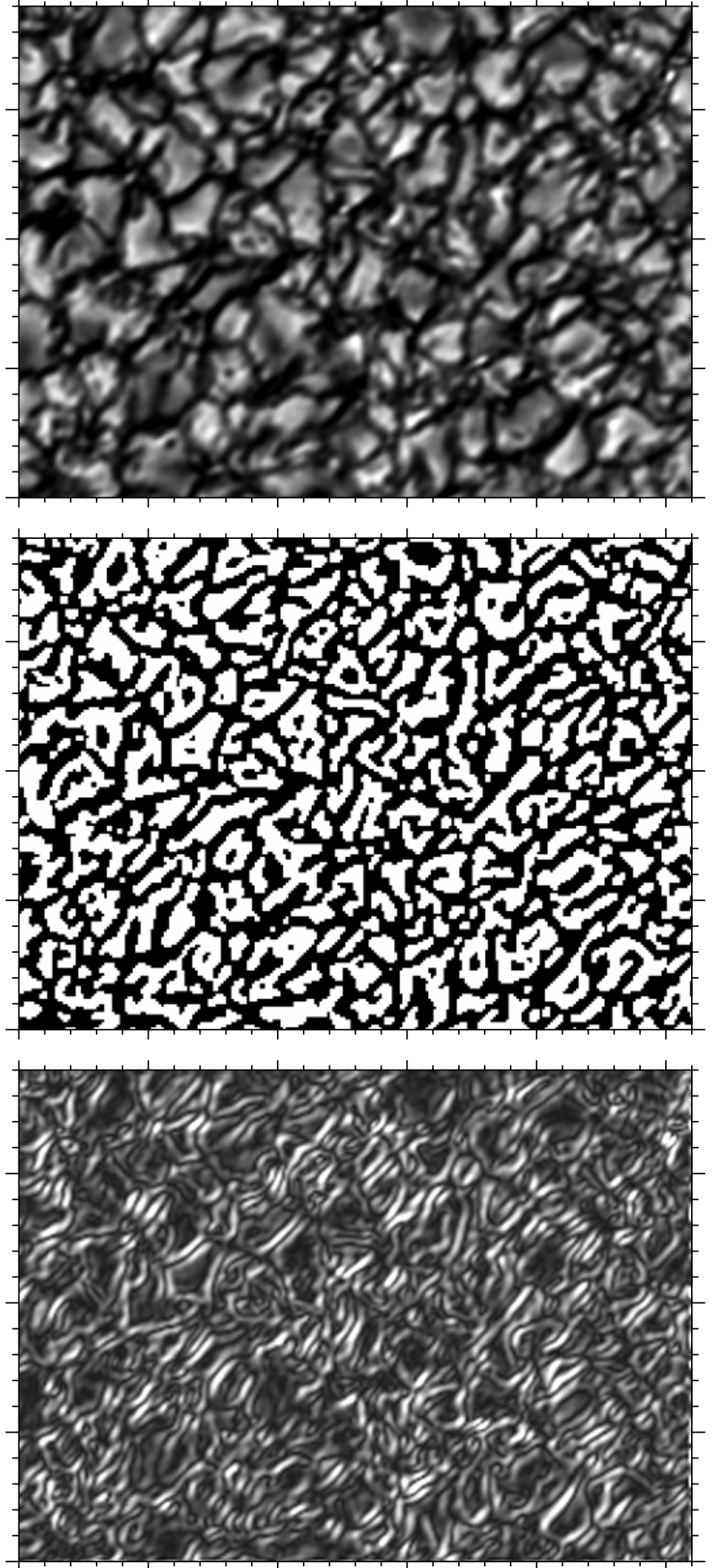

Fig. 1. Broad band image (upper panel) and granular areas obtained with the Fourier segmentation algorithm (middle panel). The lower panel shows absolute values of the horizontal intensity gradient in the broad band image. Tickmarks are at distances of $1^{\prime \prime}$.

in the velocity maps (see Sect. 2) - but the curves do not have exact Gaussian shapes as found e.g. by Keil \& Canfield (1978). The slopes towards negative velocities are significantly steeper than towards positive velocities. The maxima of the curves are at $-125 \mathrm{~m} \mathrm{~s}^{-1}$ (for $v_{0}$ ) and at $-145 \mathrm{~m} \mathrm{~s}^{-1}$ (for $v_{50}$ ), 

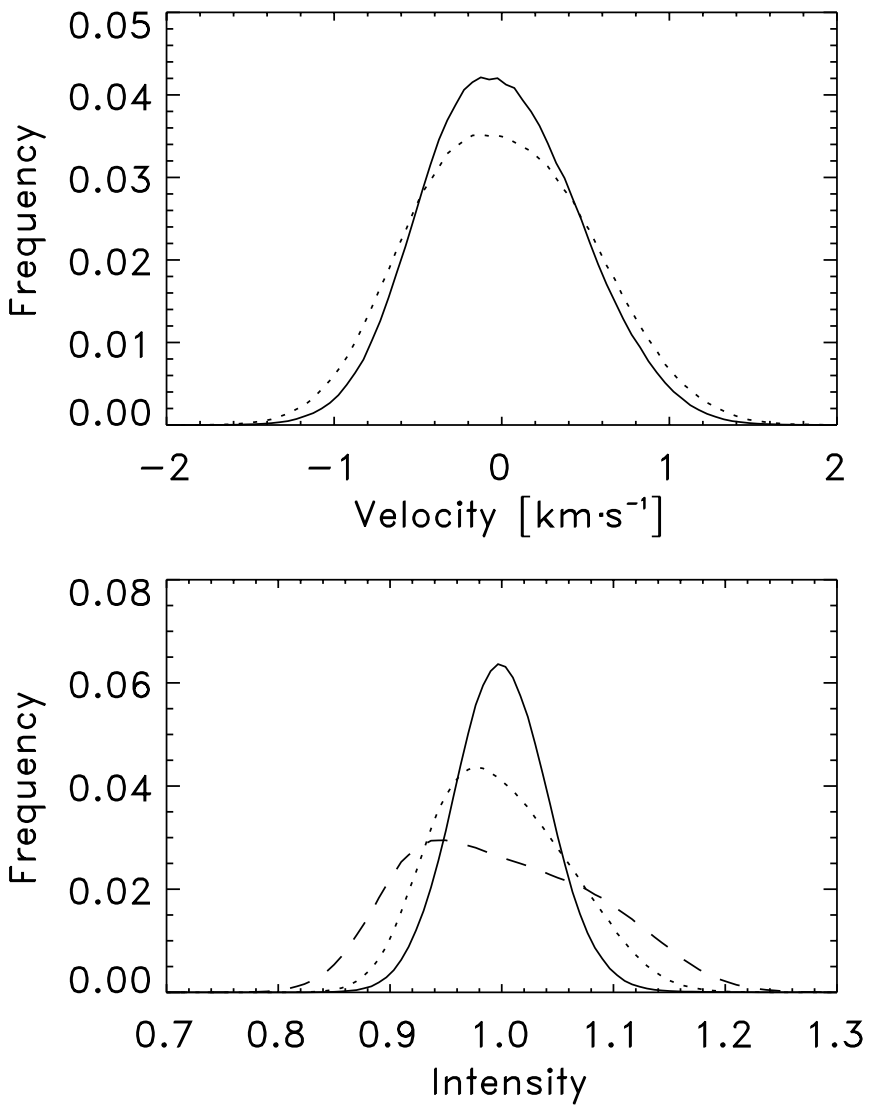

Fig. 2. Distribution of velocities and intensities in the data cubes. Solid lines correspond to the maps $v_{0}$ and $I_{0}$, dotted curves correspond to $v_{50}$ and $I_{50}$, respectively. The dashed curve in the lower panel denotes the intensity distribution in the broad band images, $I_{\mathrm{BB}}$. All curves have been normalized by dividing by the total number of pixels in the time series.

respectively. As already shown in Hirzberger et al. (2001) the velocity fluctuations exhibit only a small variation with photospheric height. The $F W H M$ of the two curves are $1.125 \mathrm{~km} \mathrm{~s}^{-1}$ (for $v_{0}$ ) and $1.380 \mathrm{~km} \mathrm{~s}^{-1}$ (for $v_{50}$ ). A further conclusion extracted from the velocity distributions is that the upflow and downflow areas are almost equal. The fractional area of positive velocities (upflows) amounts to $48.6 \%$ (for $v_{0}$ ) and to $49.0 \%$ (for $v_{50}$ ). Both values are rather close to the fractional granular area detected by the applied image segmentation algorithm (see Sect. 3 above).

The distribution functions of the intensities (lower panel of Fig. 2) show considerable variations with photospheric height. The curve corresponding to $I_{0}$ is almost symmetrical with a maximum at 0.998 and a $F W H M$ of 0.098 . In deeper photospheric levels the curves become broader - i.e. the rms contrasts are increasing - and the maxima of the curves are shifted to lower values. For $I_{50}$ the maximum of the distribution function is located at $0.978(F W H M=0.149)$ and that for $I_{\mathrm{BB}}$ is at $0.948(F W H M=0.232)$. The detected asymmetries of the distribution functions can also be seen in results from numerical simulations (Stein \& Nordlund 1998) and do not imply that the ratios of areas brighter and fainter than the average values have to deviate significantly from one. The fractions of areas brighter than the mean intensities are $49.4 \%$ (for $I_{0}$ ), $47.1 \%$ (for $I_{50}$ ), and $47.0 \%$ (for $I_{\mathrm{BB}}$ ).

\subsection{Structural parameters}

An adequate method for describing shapes of structures is the calculation of structural parameters. The distributions of four such structural parameters are plotted in Fig. 3. They have been defined, according to Stoyan \& Stoyan (1992), as follows:

The area-perimeter factor, $f_{\mathrm{AP}}$, denotes the ratio of a perimeter calculated from the area, $A$, of the structure assuming a circular shape and the actual perimeter, $P$.

$f_{\mathrm{AP}}=4 \pi A / P^{2}$

$f_{\mathrm{AP}}$ is closely related to a fractal dimension, $D$, which can be derived from the area-perimeter relation of a sample of structures and which is frequently applied to solar granulation (see e.g. Roudier \& Muller 1987; Hirzberger et al. 1997; Bovelet \& Wiehr 2001).

The circularity factor, $f_{\mathrm{C}}$, denotes the ratio of a diameter calculated from the area of the structure assuming circularity and the so-called maximum Feret-diameter, $l_{\mathrm{F}}$, which corresponds to the maximum distance of two points on the boundary of the analyzed structure.

$f_{\mathrm{C}}=\left(\frac{4 A}{l_{\mathrm{F}}^{2} \pi}\right)^{1 / 2}$

The elongation factor, $f_{\mathrm{L}}$, is defined by the ratio of the maximum Feret-diameter, $l_{\mathrm{F}}$, and the maximum extension, $w_{\mathrm{F}}$, of the structure perpendicular to $l_{\mathrm{F}}$.

$f_{\mathrm{L}}=w_{\mathrm{F}} / l_{\mathrm{F}}$.

The ellipticity factor, $f_{\mathrm{E}}$, denotes the ratio of the two semi-axes, $a$ and $b$, of an ellipse approximating the shape of the structure.

$f_{\mathrm{E}}=b / a$.

The approximation of the ellipse can be carried out by the socalled area-perimeter method, i.e.:

$a=\xi+\left[\xi^{2}-\frac{A}{\pi}\right]^{1 / 2} \quad$ with $\quad \xi=\frac{1}{3}\left[\left(\frac{A}{\pi}\right)^{1 / 2}+\frac{P}{\pi}\right]$

and

$b=\frac{A}{\pi a}$.

Figure 3 shows the distribution of the four previously defined structural parameters calculated for granules and granular cells. The distributions corresponding to the granules are generally broader than those for the granular cells. Especially the distributions of $f_{\mathrm{AP}}$ exhibit completely different shapes. The curve for the granular cells shows almost a Gaussian distribution around 0.5 reflecting that the cells have mainly regular shapes. Their perimeters are by a factor $\sqrt{2}$ longer than expected for a circle. The distribution for the granules has its maximum at $f_{\mathrm{AP}}=0.15$ and a long tail up to values of $f_{\mathrm{AP}}=0.8$. 

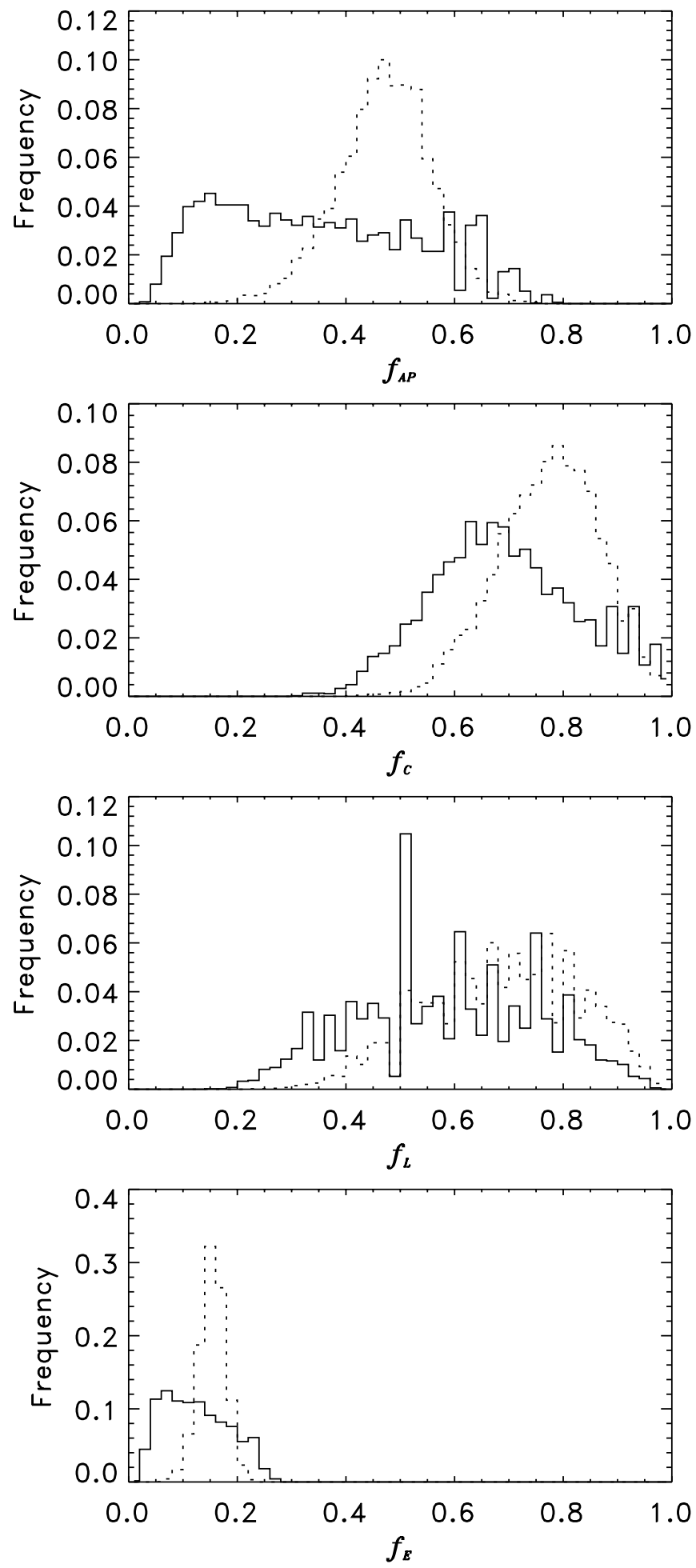

Fig. 3. Distribution functions of four structural parameters for granules (solid curves) and granular cells (dotted curves). The ordinates indicate the occurrence relative to the total number of granules in the analyzed sample.

Hence, most of the granules have extremely complex shapes but, however, a quite large number of them - mainly very small granules whose perimeters might be artificially shortened by the finite pixel size of the data - are rather regularly shaped.

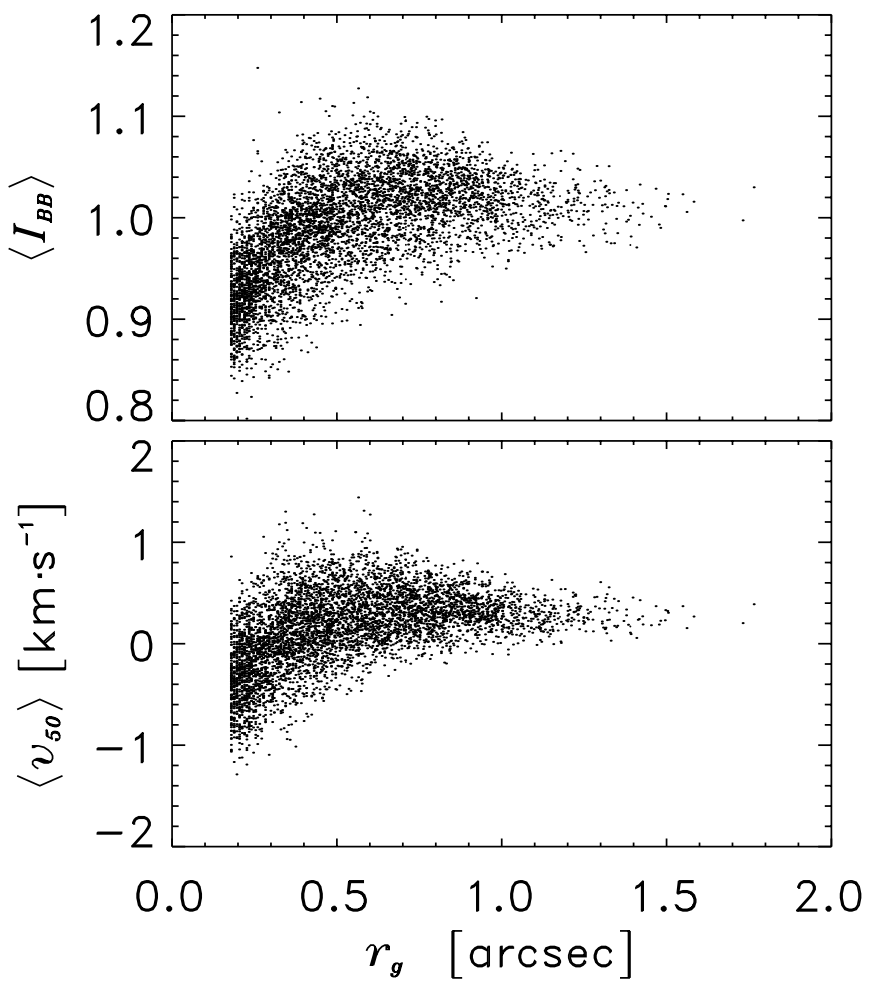

Fig. 4. Average broad band intensities $\left(\left\langle I_{\mathrm{BB}}\right\rangle\right.$, upper panel) and velocities $\left(\left\langle v_{50}\right\rangle\right.$, lower panel) for the 5509 detected granules vs. granular radius, $r_{\mathrm{g}}$. The abrupt edge for granular radii $r_{\mathrm{g}}<0$ ' 17 appears since granules with areas $A_{\mathrm{g}} \leq 9$ pixel have been removed from the sample.

For $f_{\mathrm{C}}$ the differences between granules and granular cells are not so large, yet still significant. Both distributions have a well-defined maximum and broad tails on both sides of it. The big difference between the distributions of $f_{\mathrm{C}}$ and $f_{\mathrm{AP}}$ for granules means that it is true that the granules have mainly very complex boundaries but their overall shapes do not deviate much from being circular.

The last statement has to be weakened when looking at the distribution functions of $f_{\mathrm{L}}$. Both curves cover almost the entire range between 0 and 1 . Clearly defined maxima are not visible although the curves converge rather fast to zero below $f_{\mathrm{L}}=0.3$ (granules) and $f_{\mathrm{L}}=0.4$ (granular cells). Hence, most granules might have overall regular shapes (large $f_{\mathrm{C}}$ ) with irregular boundaries (leading to a low $f_{\mathrm{AP}}$ ) but their overall shapes are significantly elongated and not circular. The peak at $f_{\mathrm{L}}=0.5$ for granules is an artefact due to the finite pixel size yielding many structures whose lengths are exactly twice as long as their widths.

The ellipticity factor $f_{\mathrm{E}}$ is extremely low for all structures, granules and granular cells. Hence, the granules do have rather elongated shapes but the shapes are far from being elliptical.

\subsection{Granular intensities and velocities}

The broad band intensities and velocities (from level $i=50 \%$ ) were averaged within each of the 5509 granules detected in the time series. Their dependence on the granular size is plotted in 
Fig. 4, in which the effective granular diameter, $r_{\mathrm{g}}$, is defined assuming circular shapes: $r_{\mathrm{g}}:=\sqrt{A_{\mathrm{g}} / \pi}$.

The average granular intensities, $\left\langle I_{\mathrm{BB}}\right\rangle$, show an increase for small radii and remain nearly constant for granules larger than approximately $r_{\mathrm{g}}=0.5$. Qualitatively this is in good agreement with former results found in Hirzberger et al. (1997) and Berrilli et al. (2002) and does also agree with numerical simulations of Gadun et al. (2000). However, the position of the elbow is slightly shifted to smaller granules as found in the above cited references. This might be a consequence of the excellent spatial resolution of the data since the image segmentation algorithm used here is the same as applied in Hirzberger et al. (1997). In the lower panel of Fig. 4 the corresponding mean granular velocities, $\left\langle v_{50}\right\rangle$, are shown. Their dependence on the granular radius agrees almost perfectly with that of the broad band intensities.

A conspicuous result to be noticed in Fig. 4 is that the distributions of both parameters, average granular intensities and velocities, do not increase monotonically with $r_{\mathrm{g}}$, i.e. the maximum values can be found in the range $0.4<r_{\mathrm{g}}<0$. 7 . This is in contrast to the distribution of the maximum granular intensities and velocities (not shown) which converge monotonically to asymptotic values (see also Figs. 12 and 13 in Berrilli et al. 2002). Therefore, granules larger than about $r_{\mathrm{g}}=0.7 \mathrm{must}$ have a rich internal structure containing very bright regions but also rather dark regions leading to a reduction of the average granular intensity. This conclusion is quite obvious when looking at a granular image, e.g. the one shown in Fig. 1. Also the development of dark centers in large granules is well known for many years. According to numerical simulations (e.g. Stein \& Nordlund 1998; Gadun et al. 2000) this arises from a pressure excess developing above large granules which reduces the upflow velocity and, consequently, the transport of thermal energy from below. The evident close similarity of the two distributions shown in Fig. 4 is, thus, well supported by theoretical models.

The average granular intensities and velocities from higher photospheric levels, $\left\langle I_{0}\right\rangle$ and $\left\langle v_{0}\right\rangle$, are plotted in Fig. 5 vs. those from lower photospheric heights. The correlation between $\left\langle v_{0}\right\rangle$ and $\left\langle v_{50}\right\rangle$ is nearly perfect and independent on the granular radius. The slope of the distribution is only slightly smaller than unity which means that the average velocities do not decrease significantly in the probed height interval. The same is valid for the maximum velocities which are not shown here.

The correlation between $\left\langle I_{0}\right\rangle$ and $\left\langle I_{50}\right\rangle$ is not that good as found for the velocities and the correlation is considerably lower for small granules than for larger ones. This is due to several reasons: (i) The overall scatter is significantly larger as for the velocities; (ii) the slope of the distributions is significantly steeper for larger granules than for smaller ones. Thus, smaller granules exhibit in average a much faster reduction of their intensity with photospheric height than larger ones; (iii) some small structures exhibit an extremely high line center intensity. Possibly, these are magnetic structures which additionally bias the correlation between $\left\langle I_{0}\right\rangle$ and $\left\langle I_{50}\right\rangle$.

It can be concluded from Fig. 5 that large granules exhibit an intensity excess also at higher photospheric levels whereas smaller granules dissolve at much lower heights. Hence, this result agrees partially with those found in Komm et al. (1991) that the typical length scale of intensity variations increases with photospheric height. For granular velocities this variation cannot be detected which contradicts the result of Komm et al. (1991).

\subsection{Granular substructure}

In Sect. 4.3 it was pointed out that (at least large) granules must have a rich internal intensity and velocity structure. Thus, an interesting question is how the intensities and velocities are distributed within the granules. As a first approximation to answer this question, the distances between the granular barycenters, $\boldsymbol{x}_{\mathrm{bc}}$, defined as

$\boldsymbol{x}_{\mathrm{bc}}=\frac{\sum_{j=1}^{N_{\mathrm{p}}} \boldsymbol{x}_{j} A_{\mathrm{p}}}{A_{\mathrm{g}}}$

and the location of the maximum intensities and maximum velocities in the granules can be computed. The quantities $N_{\mathrm{p}}$ and $A_{\mathrm{p}}$ denote the number of pixels belonging to a granule and the area of one pixel, respectively. In Fig. 6 the dependences of the relative - i.e. normalized to the granular radius, $r_{\mathrm{g}}-$ distances of the maximum granular broad band intensities, $I_{\mathrm{BB}, \max }$, and velocities, $v_{50, \max }$, from the barycenters of the granules on the granular radius are shown.

It is evident from the distributions plotted in Fig. 6 that for small granules the maximum intensities as well as the maximum velocities are located much closer to the granule barycenters as for larger ones. The dependences on the granular radius show nearly linear trends. This result confirms statistically the results of e.g. Nesis et al. (1992) or Krieg et al. (2000) who claimed that the maximum granular intensities and velocities tend to be located close to the granule boundaries although this claim seems to be valid only for very large granules. This result also agrees well with numerical models of Rast (1995) in which the fastest and brightest granular regions are locations of displaced material when fast downdrafts develop. Therefore, the fastest and brightest granular regions are located close to the intergranular lanes, i.e. at the granular boundaries.

The very large relative distances, $\Delta d_{\mathrm{BB}} / r_{\mathrm{g}}>1$ and $\Delta d_{\mathrm{v}, 50} / r_{\mathrm{g}}>1$ which can be found in Fig. 6 do not mean that the brightest pixels are located outside the granule, i.e. that the segmentation algorithm is not working properly. This is rather due to the very complex granular shapes, e.g. corresponding to elongation factors far below $f_{\mathrm{L}}=0.5$ (see Fig. 3).

The location of pixels with maximum intensities and velocities has low statistical significance in cases where granules have areas of several hundred pixels. Moreover, the distribution shown in the middle panel of Fig. 6 seems to be also biased by a residual distortion between broad band images and velocity maps. This can be seen in the region 0. . $2<r_{\mathrm{g}}<0$. . 4 and $1<\Delta d_{\mathrm{v}, 50} / r_{\mathrm{g}}<2$ where many displacements are found. For these tiny granules the detected shapes from the broad band images might be somewhat distorted with respect to the velocity maps. This is a consequence of the limited spatial resolution of the narrow band data which is in the range of $0 . ' 4$, and thus approximately 4 pixels (see Hirzberger et al. 2001). 

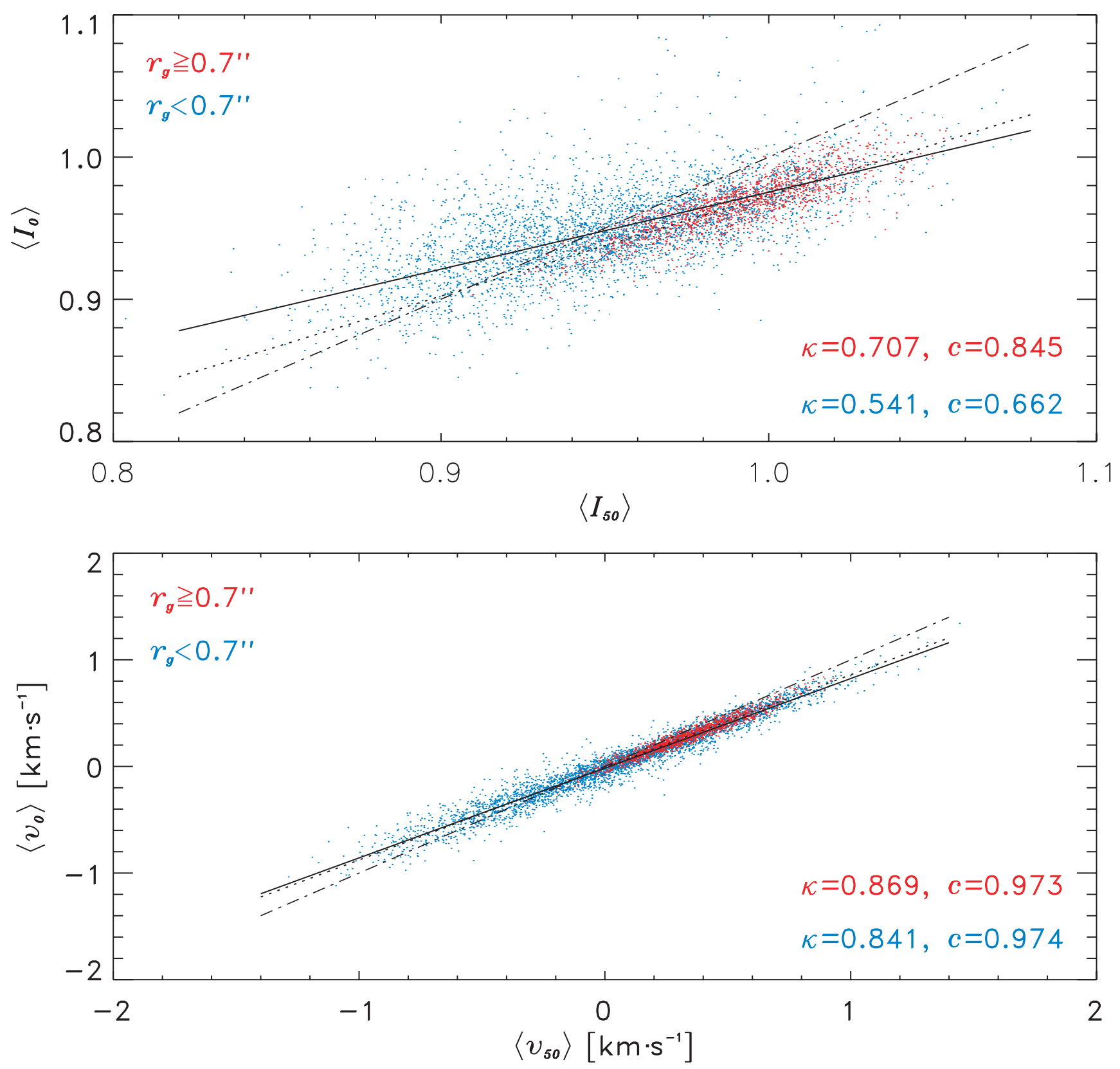

Fig. 5. Upper panel: Average granular line center intensities, $\left\langle I_{0}\right\rangle$, vs. those from level $i=50 \%$ (line wings), $\left\langle I_{50}\right\rangle$; lower panel: average granular velocities, $\left\langle v_{0}\right\rangle$ vs. $\left\langle v_{50}\right\rangle$. Blue dots denote granules smaller than $r_{\mathrm{g}}=0$ '.7, red dots denote granules with $r_{\mathrm{g}} \geq 0$.'7. The overplotted solid lines represent linear fits to the granules with $r_{\mathrm{g}} \geq 0$.'7, dotted lines are fits to granules with $r_{\mathrm{g}}<0.7 . \kappa$ and $c$ are slopes of linear regression fits and their corresponding correlations. Dash-dotted curves represent the medians, $\kappa=1$.

To overcome this problem and to increase the statistical significance of the measured intensity and velocity structure within the granules, "inertial radii", $r_{\Theta}$, can be defined from the moment of inertia around a vertical axis through the granular barycenters. For convenience, i.e. to include also the structure of the intergranular lanes surrounding the granules, $r_{\Theta}$ has been computed from the entire granular cells including granular and intergranular regions as defined in Sect. 3.

$r_{\Theta}=\left(\frac{\sum_{j=1}^{N_{\mathrm{p}}} d_{j}^{2} \rho\left(d_{j}\right) A_{\mathrm{p}}}{M}\right)^{1 / 2}$ where

$M=\sum_{j=1}^{N_{\mathrm{p}}} \rho\left(d_{j}\right) A_{\mathrm{p}}$

denotes the granular "mass" and the quantities $d_{j}$ and $\rho\left(d_{j}\right)$ are the distance of the $j$ th pixel from the granule barycenter and the local density (i.e. intensity, velocity, etc.) measured at the $j$ th pixel, respectively. Here $N_{\mathrm{p}}$ denotes the number of pixels belonging to a granular cell.

Figure 7 (upper panel) shows area inertial radii, $r_{\mathrm{A}}$, i.e. assuming that the density $\rho$ is constant in the granular cell, for the 5509 detected granules vs. the granule radius. The absolute $r_{\mathrm{A}}$ 


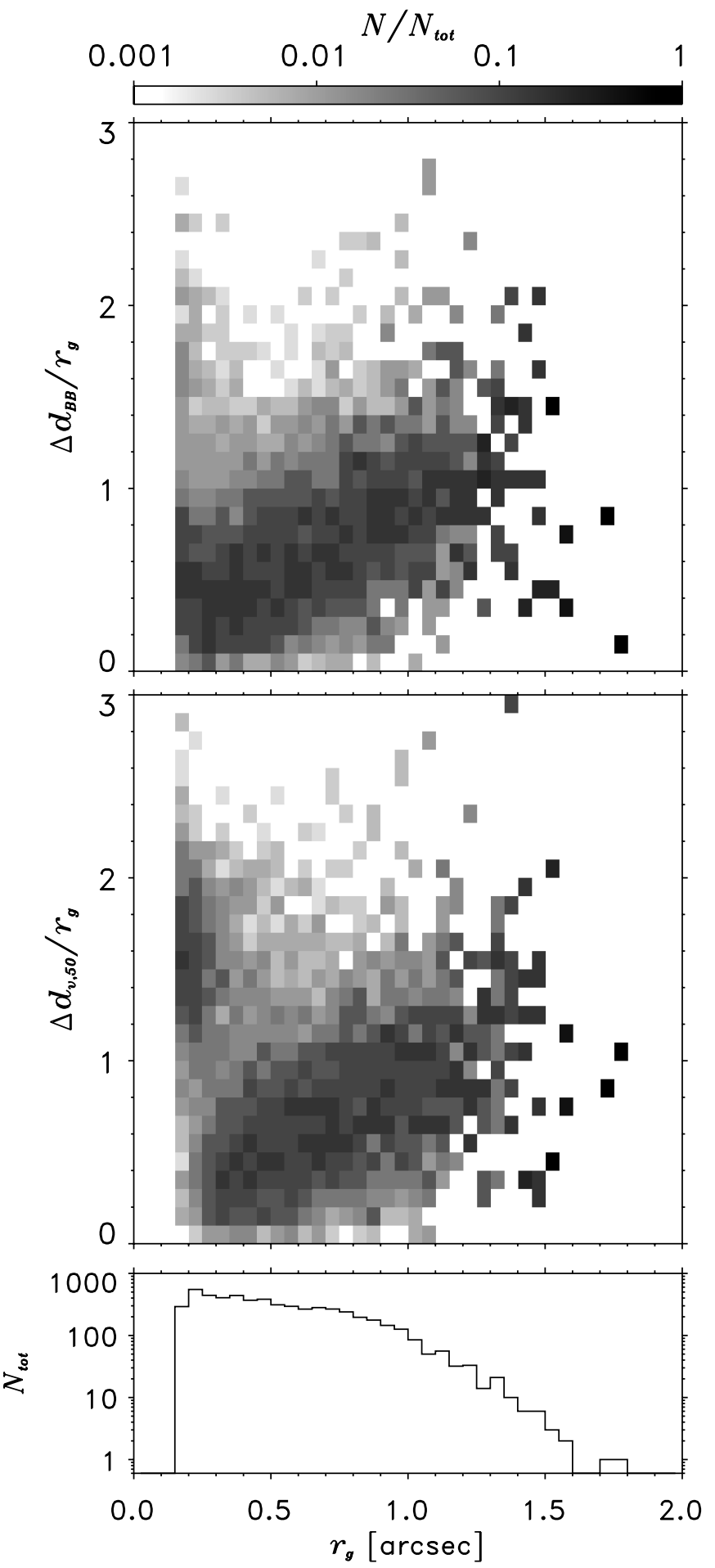

Fig. 6. Two-dimensional distributions of relative distances of maximum broad band intensities $\left(\Delta d_{\mathrm{BB}} / r_{\mathrm{g}}\right.$, upper panel) and velocities $\left(\Delta d_{\mathrm{v}, 50} / r_{\mathrm{g}}\right.$, middle panel) from the granule barycenters. The distributions have been normalized to the total number of granules in the corresponding radius interval, $N_{\text {tot }}$, taken from the histogram in the lower panel.

have been normalized to the cell radii, $r_{\mathrm{c}}:=\sqrt{A_{\mathrm{c}} / \pi}$. The distribution shows no clear trend. For most of the small granules the relative area inertial radius, $r_{\mathrm{A}} / r_{\mathrm{c}}$, lies in the range between 0.8 and 1 . For the sake of comparison, the inertial radius (assuming
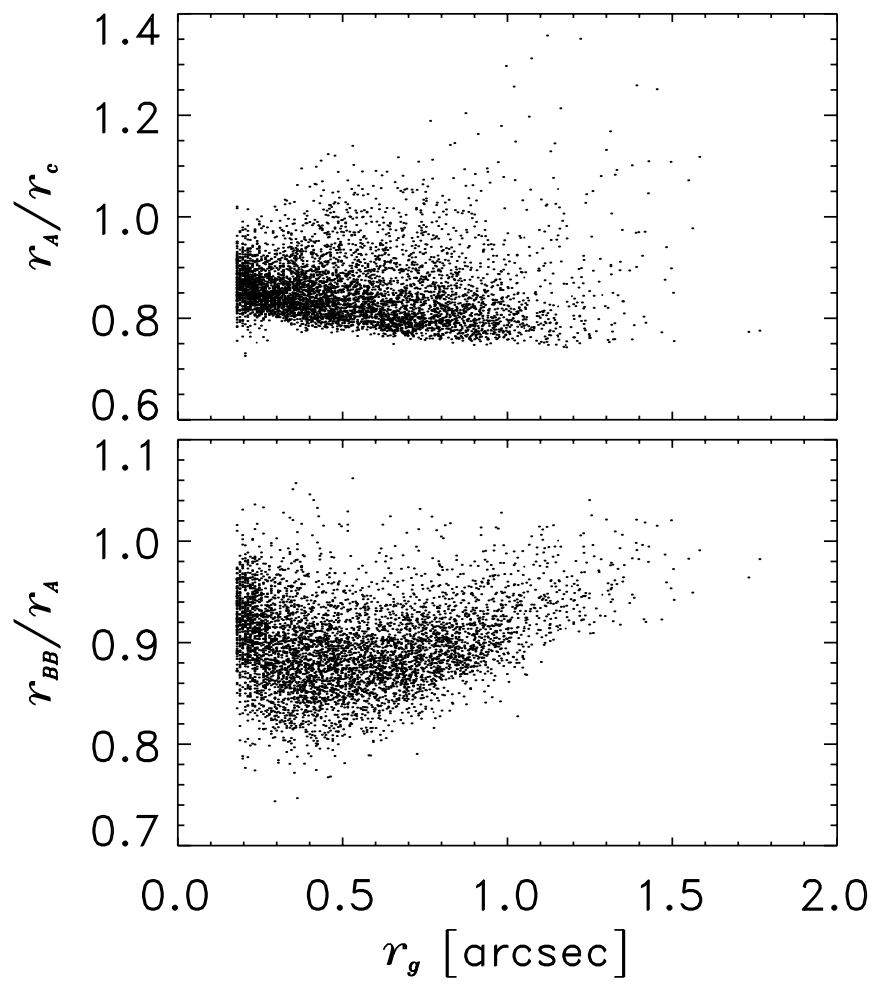

Fig. 7. Upper panel: relative area inertial radii of the granular cells vs. granular radius; lower panel: ratios between the inertial radii derived from the broad band intensity and the area inertial radii vs. granule radius.

constant density) for a circle with radius $R$ is $r_{\mathrm{A}} / R=1 / \sqrt{2}$ and for a rectangle with a side ratio of $1 / 3$ the relative area inertial radius is $\sqrt{10 \pi / 36} \approx 0.934$. For larger structures the $r_{\mathrm{A}} / r_{\mathrm{c}}$ scatter is in a range between 0.75 and 1.4 although a slight tendency for a general decrease of $r_{\mathrm{A}} / r_{\mathrm{c}}$ with $r_{\mathrm{g}}$ can be detected. This means that most of the large granular cells are more roundish than smaller ones - this might be slightly biased by the finite pixel size - but some of them (those with $r_{\mathrm{A}} / r_{\mathrm{c}}>1$ ) must have very elongated shapes, too.

The lower panel of Fig. 7 shows ratios between the broad band inertial radii of the granular cells, $r_{\mathrm{BB}}$, and the area inertial radii. The $r_{\mathrm{BB}}$ have been calculated setting $\rho=I_{\mathrm{BB}}$ in Eq. (6). For very small granules with $r_{\mathrm{g}}<0$.' 4 the ratio increases with decreasing size, achieving values larger than 0.9 for the tiniest structures. Hence, these granules must have an almost homogeneous intensity structure $\left(r_{\mathrm{BB}}=r_{\mathrm{A}}\right.$ for constant $\left.I_{\mathrm{BB}}\right)$. Of course the ratio does not achieve unity because the intergranular lanes around these small granules reduces $r_{\mathrm{BB}}$ compared to $r_{\mathrm{A}}$. For granules in the range $00^{\prime} 4<r_{\mathrm{g}}<0.7$ the ratio of inertial radii exhibits a clear minimum. For these granules bright intensity maxima must exist close to their barycenters. For granules larger than $r_{\mathrm{g}}=0.7$ the ratio increases with the granule radius, i.e. the larger the granules are the closer the brightest regions are situated to the granule boundaries. This result is in good agreement with Fig. 6 but has a much higher statistical significance.

In Fig. 8 intensity inertial radii computed from the broad band images and the intensity maps together with velocity 


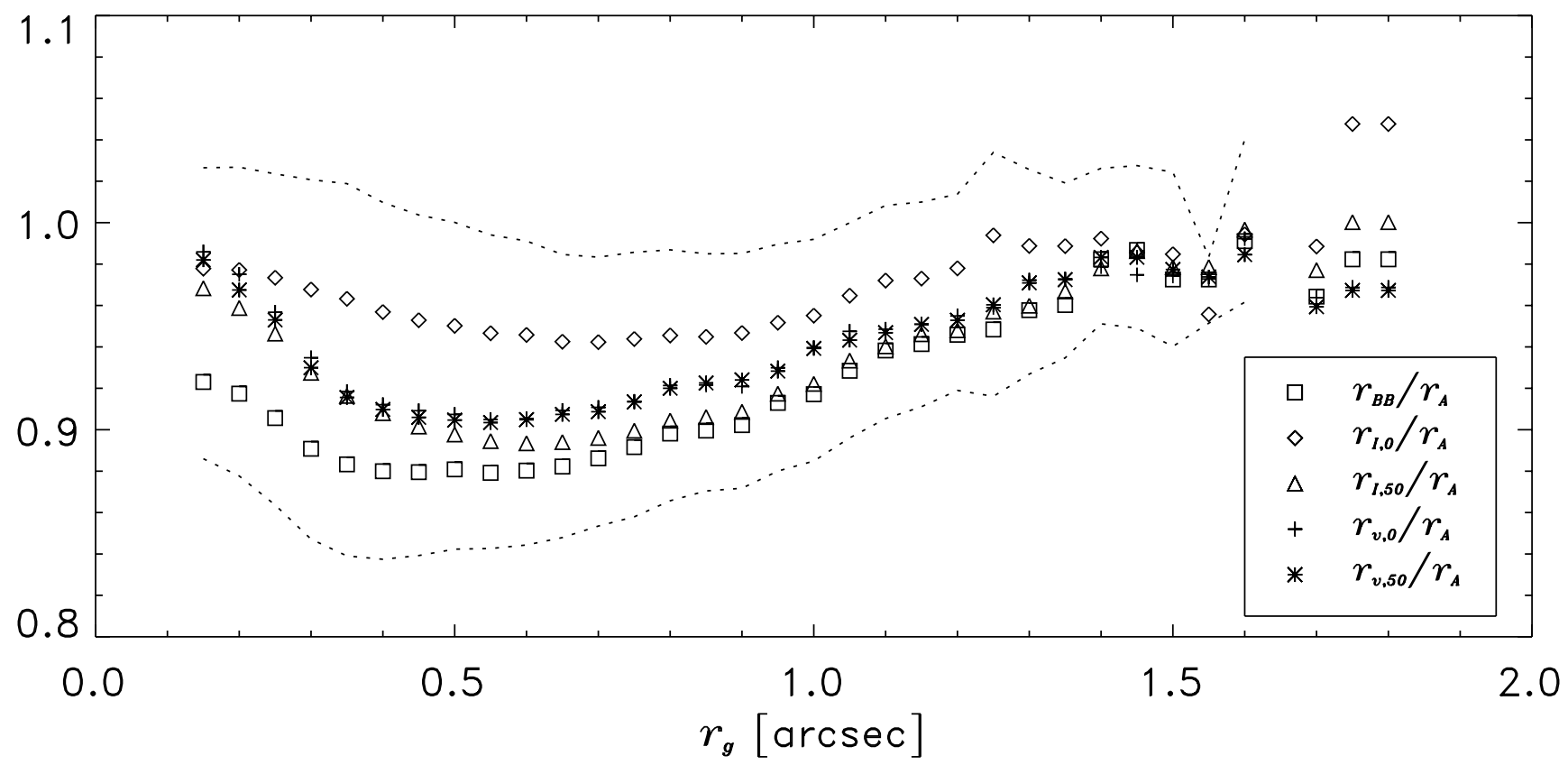

Fig. 8. Averaged ratios of the inertial radii derived from the intensity and velocity maps and the area inertial radii. The average has been carried out in overlapping bins of 0 !' 1 width. The dashed curves represent standard deviations (error bars) in each bin of the plot; the lower one has been calculated for $r_{\mathrm{BB}} / r_{\mathrm{A}}$ (squares), the upper one for $r_{\mathrm{I}, 0} / r_{\mathrm{A}}$ (diamonds).

inertial radii computed from the velocity maps are plotted vs. $r_{\mathrm{g}}$. The absolute inertial radii, $r_{\mathrm{BB}}, r_{\mathrm{I}, 0}, r_{\mathrm{I}, 50}, r_{\mathrm{v}, 0}$, and $r_{\mathrm{v}, 50}$ have been normalized to the area inertial radius $r_{\mathrm{A}}$ of each cell. For the sake of an easier representation the resulting ratios have been averaged in overlapping bins of 0 !' 1 width (moving window method). This method using overlapping bins has the advantage that the averaged curves additionally become effectively smoothed. Qualitatively, all the curves plotted in Fig. 8 have the same shapes, i.e. they are close to one for the very smallest and very largest granules and have a minimum at intermediate granular radii. The two curves calculated from the velocity maps $\left(v_{0}\right.$ and $\left.v_{50}\right)$ are almost identical, i.e. the velocity structure does not change in the probed photospheric height interval. The curve derived from $I_{0}$ deviates significantly from the others: (i) the minimum is shifted to larger granules $\left(r_{\mathrm{g}} \approx 0\right.$.'7) and (ii) the minimum is much shallower than those of the other three curves. The shift of the minimum can be explained by the fact that the intensity excess of smaller granules dissolves at lower photospheric heights than that of larger ones (see also Fig. 5). The shallow minimum is due to the much lower rms intensity fluctuations measured in $I_{0}$ compared to those in $I_{50}$ and $I_{\mathrm{BB}}$ (see Hirzberger et al. 2001).

For the very smallest granules the ratio of the inertial radii approaches almost one for the curves derived from $I_{0}, I_{50}, v_{0}$, and $v_{50}$, respectively, whereas the curve corresponding to $I_{\mathrm{BB}}$ reaches only 0.92 . This might be explained by a residual distortion of the intensity and velocity maps compared to the broad band images. Hence, the granular cells detected in the broad band images are not exactly co-aligned with the corresponding granules in the intensity and velocity maps. However, this effect becomes crucial only for the very smallest structures with $r_{\mathrm{g}}<0$ '.2 because for these structures the expected residual

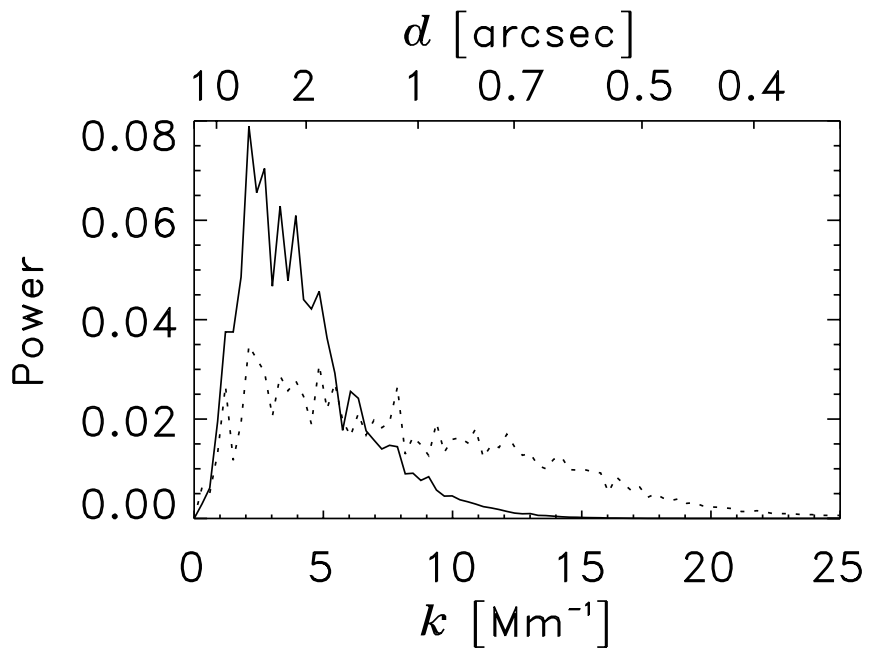

Fig. 9. Two-dimensional - azimuthally averaged along circles of constant wavenumbers $k=\sqrt{k_{x}^{2}+k_{y}^{2}}$ - power spectra of the broad band image (solid line) and the gradient image (dotted line) displayed in Fig. 1.

distortion - which is in the range of the expected spatial resolution of the narrow band data, i.e., approximately $0{ }^{\prime} 4$ - is in the range of the sizes of the granules.

\subsection{Horizontal intensity and velocity gradients}

The result from the previous section - highest intensities and velocities are located close to the granular boundaries - implies a sharp transition of both parameters from the granules to the intergranular lanes, i.e. high horizontal intensity and velocity 

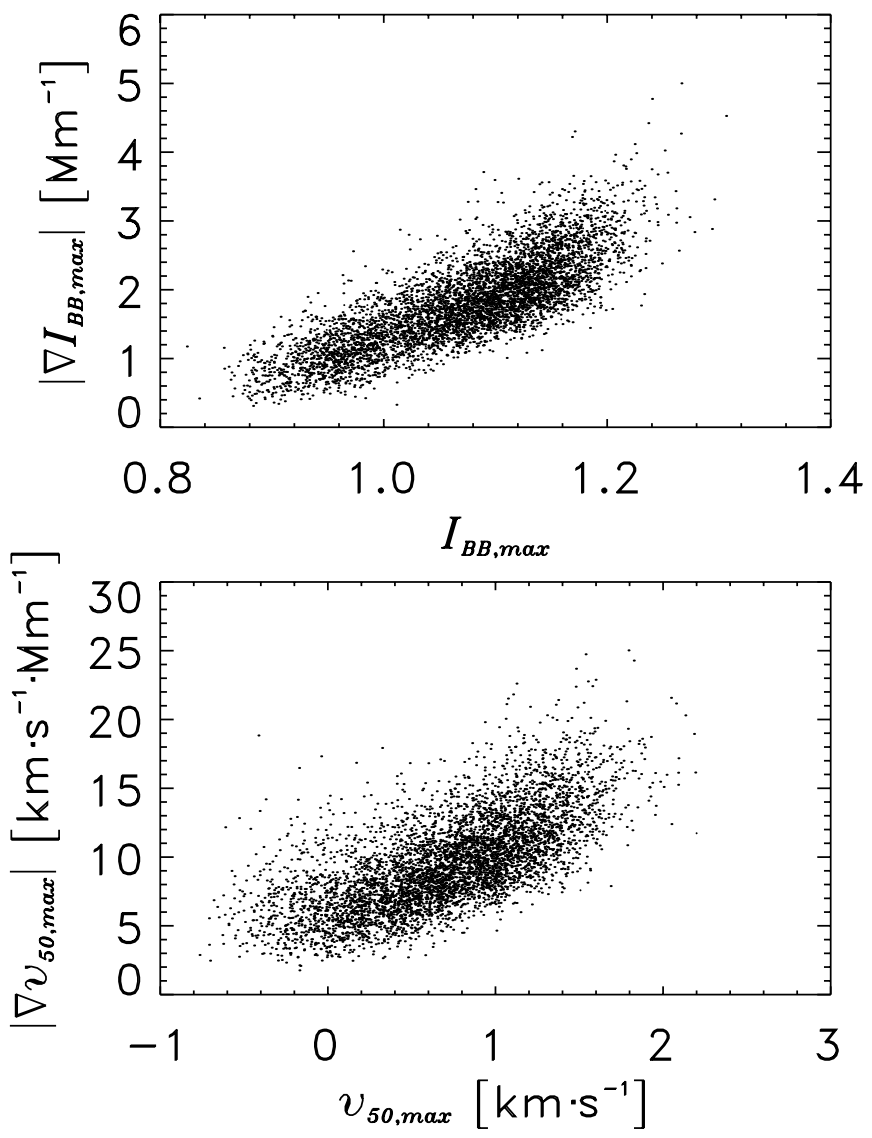

Fig. 10. Upper panel: maximum gradient of the broad band intensity of the 5509 granules vs. maximum broad band intensities in the granules; lower panel: maximum gradient of the velocity (at $i=50 \%$ ) vs. maximum velocity.

gradients. In the lower panel of Fig. 1 an example of the broad band intensity gradient, $\left|\nabla I_{\mathrm{BB}}\right|=\left[\left(\partial I_{\mathrm{BB}} / \partial x\right)^{2}+\left(\partial I_{\mathrm{BB}} / \partial y\right)^{2}\right]^{1 / 2}$, is displayed. The derivatives have been calculated using a three point Lagrangian interpolation algorithm. The structures visible in this image coincide, as expected, well with the contours of the granules. At first glance it seems that all visible structures have the same width of about $0{ }^{\prime} .5$ which means that the width of the transition zone between granules and intergranular lanes is independent on the size and the intensity of the granules.

Figure 9 shows power spectra of the broad band image and of the gradient map displayed in Fig. 1. For structures with diameters, $d<2^{\prime \prime}$, the power spectrum of the broad band image falls nearly exponentially to zero (for a discussion of the power laws of granulation images see e.g. Espagnet et al. 1993; Hirzberger et al. 1997; Nordlund et al. 1997) whereas the power spectrum of the gradient image shows a linear decrease down to a wavenumber of approximately $k=17 \mathrm{Mm}^{-1}(d=0.51)$. Since the structures visible in the gradient image (Fig. 1) are mainly thin and elongated, this result can be interpreted such that it is dominated by structures with a maximum length of approximately $4^{\prime \prime}\left(k=2.17 \mathrm{Mm}^{-1}\right)$ and with a minimum extension or typical width of 0.5 .

In the upper panel of Fig. 10 the maximum gradient of the broad band intensity in each of the 5509 granular cells (granules plus surrounding intergranular lanes) vs. the maximum broad band intensity in each granular cell is plotted. In the lower panel of Fig. 10 the maximum gradients of the velocities $(i=50 \%)$ vs. the maximum velocities in the granules are shown. Both plots exhibit a clear and nearly linear trend although the lower one is tainted with somewhat higher scatter resulting in a flattening of the trend for structures with maximum velocities below zero. These latter structures do have maximum intensities below one, i.e. they are small structures which seem to be slightly affected by some residual noise in the velocity maps.

The appearance of the linear trends in Fig. 10 means that brighter granules and granules containing faster upflows show a steeper drop of intensity and velocity towards the intergranular lanes than fainter ones. This follows from the fact that (i) the brighter the granules are the closer the location of the maximum intensities and velocities is situated to the granular boundary and (ii) that the width of the structures found in the gradient images (e.g. the one in Fig. 1) is independent on the granular size or intensity. However, this result does not mean that the brightest granules must be surrounded by the darkest intergranular lanes. Plotting maximum granular broad band intensities vs. minimum intergranular broad band intensities (not shown) exhibits a slight negative trend but with a correlation of only -0.21 . The correlation between maximum granular velocities and minimum intergranular velocities is, with -0.18 (for $i=0 \%$ ) and with -0.16 (for $i=50 \%$ ), even weaker.

\subsection{Coherence analysis}

In the present data the time interval between two images is $70 \mathrm{~s}$, which is in the range of the lifetimes of small granules (see Hirzberger et al. 1999). Hence, for studying the temporal evolution of the granulation pattern a direct tracking of individual structures is not possible. An alternative attempt is shown in Fig. 11. In this figure, the temporal variation of the coherence spectra of broad band images and line center intensity maps are plotted. The coherence spectrum, $C(k)$, of two images, $F(x, y)$ and $G(x, y)$, is defined as

$C(k)=\frac{\left|\left\langle\tilde{F}\left(k_{x}, k_{y}\right) \cdot \tilde{G}^{*}\left(k_{x}, k_{y}\right)\right\rangle\right|}{\left\langle\left|\tilde{F}\left(k_{x}, k_{y}\right) \cdot \tilde{G}^{*}\left(k_{x}, k_{y}\right)\right|\right\rangle}$,

where the : denotes Fourier transformed quantities depending on the wavenumbers $k_{x}$ and $k_{y}$. The brackets denote averages over a suitable region, e.g. in circles of constant $k=\sqrt{k_{x}^{2}+k_{y}^{2}}$ (azimuthal average).

The columns in the images shown in Fig. 11 denote averages of coherence spectra, $C\left(t_{m}, k\right)$, from images, $F_{l}$ and $F_{l+m}$, separated by a constant time interval $t_{m}=m \cdot \Delta t$ :

$C\left(t_{m}, k\right)=\frac{\sum_{l=0}^{N-m-1} C\left(F_{l}, F_{l+m}\right)}{N-m}, \quad m=0, \ldots, N-1$

where $\Delta t=70 \mathrm{~s}$ is the time interval between two images and $N=36$ is the number of images in the time series. The columns corresponding to $t_{m}=0$ (of the plots in Fig. 11) denote 

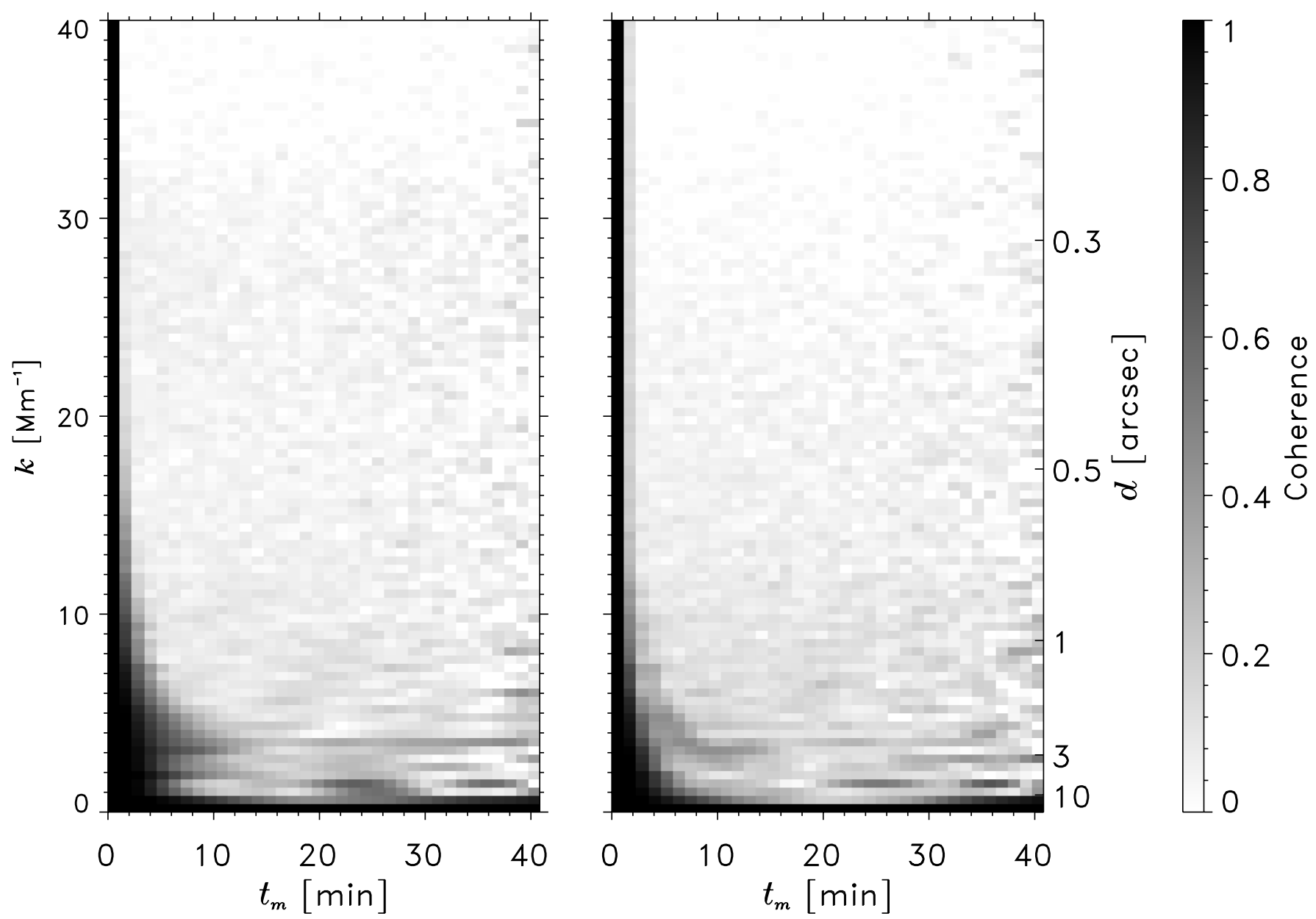

Fig. 11. Averaged coherence spectra of broad band images (left panel) and of line core intensity maps (right panel). The parameter $d=2 \pi / k$ denotes a spatial wavelength of structures.

averaged coherence spectra when correlating images with themselves which is equal to unity for all wavenumbers, $k$. The second columns $\left(t_{m}=70 \mathrm{~s}\right)$ represent coherence spectra when correlating images with the subsequent ones and so on.

Figure 11, hence, shows the temporal variation of the coherence of the granulation pattern in dependence on the structural sizes. In the left panel (broad band data) the coherence is high in the region around $k=5 \mathrm{Mm}^{-1}\left(1^{\prime \prime} .73\right)$ and for $t_{m} \lesssim 10 \mathrm{~min}$ which denotes typical sizes and lifetimes of large granules. For smaller structures $\left(5 \mathrm{Mm}^{-1}<k<20 \mathrm{Mm}^{-1}\right)$ the time interval of high coherences drops quickly to zero. For smaller wavenumbers the same is valid but the coherence shows additional peaks at $t_{m} \approx 23 \mathrm{~min}$ and at $t_{m} \approx 36 \mathrm{~min}$ for $k=1.6 \mathrm{Mm}^{-1}$ and at $t_{m} \approx 40 \mathrm{~min}$ for $k=0.7 \mathrm{Mm}^{-1}$. A slight increase of the coherence is also visible at $k=3.8 \mathrm{Mm}^{-1}$ and $30 \mathrm{~min}<t_{m}<40 \mathrm{~min}$. These secondary peaks might be resulting from large and recurrent exploding granules first detected by Carlier et al. (1968) or from strong positive divergences (SPDs) found by Rieutord et al. (2000). Meso- and supergranular structures are expected to have longer lifetimes than the time intervals between these secondary peaks. Moreover, they should not be visible that clearly in the broad band data although it has to be assumed that the positions where recurrent granules are situated are somehow related to those large-scale flow fields (see e.g. Oda 1984; Title et al. 1989). Yet, a detailed study of these structures visible in the very low $k$-range is not the aim of the present work because of the limited field of view yielding a rather low wavenumber resolution in the range below $k=3 \mathrm{Mm}^{-1}$.

The coherence spectra of the line core intensity maps (right panel of Fig. 11) show a gap in the region $4 \mathrm{~min}<t_{m}<10 \mathrm{~min}$ and $1 \mathrm{Mm}^{-1}<k<3 \mathrm{Mm}^{-1}$. It can be concluded from this result that granules are almost completely dissolved at this photospheric height $(320 \mathrm{~km})$. Smaller structures are clearly visible and seem to have quite long lifetimes of more than $10 \mathrm{~min}$. These structures cannot be granules because small ones should dissolve at much lower photospheric heights than larger ones. Possibly, the origin of the high coherence in that region are magnetic structures which become bright in high photospheric levels. In the line core maps a few of these structures are visible but their number is very small (see also Fig. 5) so that they do not considerably bias the statistics carried out in the previous sections.

The secondary peaks at $k=1.6 \mathrm{Mm}^{-1}\left(t_{m} \approx 23 \mathrm{~min}\right.$ and $\left.t_{m} \approx 36 \mathrm{~min}\right)$ and $k=0.7 \mathrm{Mm}^{-1}\left(t_{m} \approx 40 \mathrm{~min}\right)$ are also visible in the line core coherence spectra but the increase of coherence at $k=3.8 \mathrm{Mm}^{-1}$ has almost disappeared. Since broad band and narrow band data are obtained from independent observations, 
the secondary peaks should represent real physical phenomena. The very largest and brightest exploding granules which might be responsible for these secondary peaks are expected to produce positive temperature and brightness excesses also at high photospheric levels (see e.g. Roudier et al. 2001). Thus, it is not surprising that they are also visible in the line core data.

It is possible to obtain lifetimes of granular features from the coherence spectra shown in Fig. 11. In Fig. $12 e$-folding times, $t_{\mathrm{e}}(k)$, i.e. the times, $t_{m}$, in which the coherences fall below $1 / e$, vs. $k$ are plotted. To overcome the relatively low temporal resolution of the analyzed data $(70 \mathrm{~s}$ cadence between two images) the coherences have been interpolated using cubic splines before calculating the $e$-folding times. The curves are nearly identical for all the used data, except the curve corresponding to the line core intensity maps deviates slightly from the others. The $e$-folding times of the remaining parameters show well defined maxima at $k \approx 3.5 \mathrm{Mm}^{-1}$ and a quite noisy behaviour for smaller $k$. This noisy character is probably caused by a combination of two effects: (i) the poor resolution in the low $k$-range and (ii) due to the large variety of phenomena in this range, e.g. large exploding granules (recurrent and non-recurring), SPDs, meso- and supergranules, etc.

In the range $k>3.5 \mathrm{Mm}^{-1}$ the $e$-folding times exhibit an almost perfect linear (in this log-log representation) decrease from $t_{\mathrm{e}}=11 \mathrm{~min}$ down to the time interval between two images in the time series is reached, which takes place at $k \approx 20 \mathrm{Mm}^{-1}$. The linearity continues slightly below $t_{\mathrm{e}}=70 \mathrm{~s}$ which is caused by the spline interpolation of the measured coherences. The inset in Fig. 12 shows once more the $e$-folding times for the broad band images (in the range $3.2 \mathrm{Mm}^{-1}<k<20.5 \mathrm{Mm}^{-1}$ ). The overplotted linear fit represents a power law of the form

$t_{\mathrm{e}} \sim k^{-\beta}$ with $\beta=1.512 \approx 3 / 2$.

The correlation of the fit amounts to $c=0.998$. Hence, the existence of a power law for granular lifetimes with increasing wavenumber has to be concluded from this result. This holds for both the granular velocity pattern and for the intensity pattern at low photospheric heights. The dissolution of the intensity pattern in the upper photosphere causes the deviation of the curve corresponding to the line core intensity maps. The peak at $k=5 \mathrm{Mm}^{-1}$ might be produced by long-lasting magnetic structures which become rather large in these high photospheric levels since the equilibrium of the (reduced) gas pressure and the magnetic pressure can be maintained only if the magnetic structures expand.

\section{Summary and conclusions}

In the present paper a statistical analysis of the intensity distribution and the vertical flow structure of quiet solar granulation has been carried out. The results show a good agreement between the broad band intensity structure and the vertical flow velocities. The latter show almost no variation within the probed photospheric height range. The intensity fluctuations depend much more on the photospheric height than the velocities. Only the very largest granules produce a positive intensity excess at a height of $320 \mathrm{~km}$ whereas smaller structures dissolve at much lower photospheric heights which is - partially - in good agreement with former results of e.g. Komm et al. (1991). In this latter study it has also been found that small scale velocity fluctuations dissolve at much lower photospheric heights than larger ones. These findings are not supported by the results obtained from the present data.

An image segmentation algorithm has been applied to separate granular and intergranular regions. The fractional granular area is about $47.7 \%$ which is much larger than that found by Roudier \& Muller (1987) but much smaller than the fractional granular area found in Bovelet \& Wiehr (2001). In the analyzed data the fractional area brighter than the mean photospheric brightness is $47 \%$ (broad band images) and the fractional area belonging to upward directed granular velocities (at $i=50 \%$ ) amounts to $49 \%$ of the total area which is in good agreement with the segmented granular area.

The segmented granular structures have quite complex shapes containing a rich internal structure. In small granules the maximum intensities and the maximum upward velocities are located close to the granular barycenters whereas in large granules the brightest parts and the maximum upward velocities are shifted towards the granular boundaries. This is in good agreement with findings of Nesis et al. (1992) and with numerical models of Rast (1995). However, Nesis et al. (1993) have found high intensities but zero or even downward directed velocities at the granular boundaries. They surmise that enhanced turbulence appears at the granular boundaries which produces the low correlation values, found by them, between intensities and velocities at the granular boundaries.

The maximum horizontal intensity and velocity gradients coincide - as expected - well with the granular boundaries. Moreover, the data exhibit a clear and nearly linear dependence of the maximum gradients on the maximum intensities and velocities, respectively. It can be concluded from this result that the widths of the transition zones between granules and intergranular lanes are independent of the intensity and the size of the granules. This conclusion can also be drawn when studying the gradient images (e.g. the one in Fig. 1) and the same conclusion holds for granular velocities.

According to numerical simulations of Rast (1995, 1999a) the intergranular lanes are formed by the development of localized downflowing plumes due to Rayleigh-Taylor instabilities. These plumes cause a displacement of matter away from the downflow channels which produce intensity and velocity maxima just at the boundaries of solar granules. The amplitude of the intensity and velocity excesses at the granular boundaries are, therefore, "... locally in response to buoyancy and pressure gradient forces induced by the downflowing fluid..." (Rast 1995). Hence, a close connection between both brightness and velocities of granules and intensity and velocity gradients has to be expected. The constant width of the transition zone between granules and intergranular lanes - which is in the range of about 0.5 - needs also an explanation. One possibility is that it is produced by lateral heat exchange due to radiative effects. Another possibility is the generation of a turbulent layer due to shear forces in the region where the sign of the vertical flow velocity is changing (see Stein \& Nordlund 1998). 


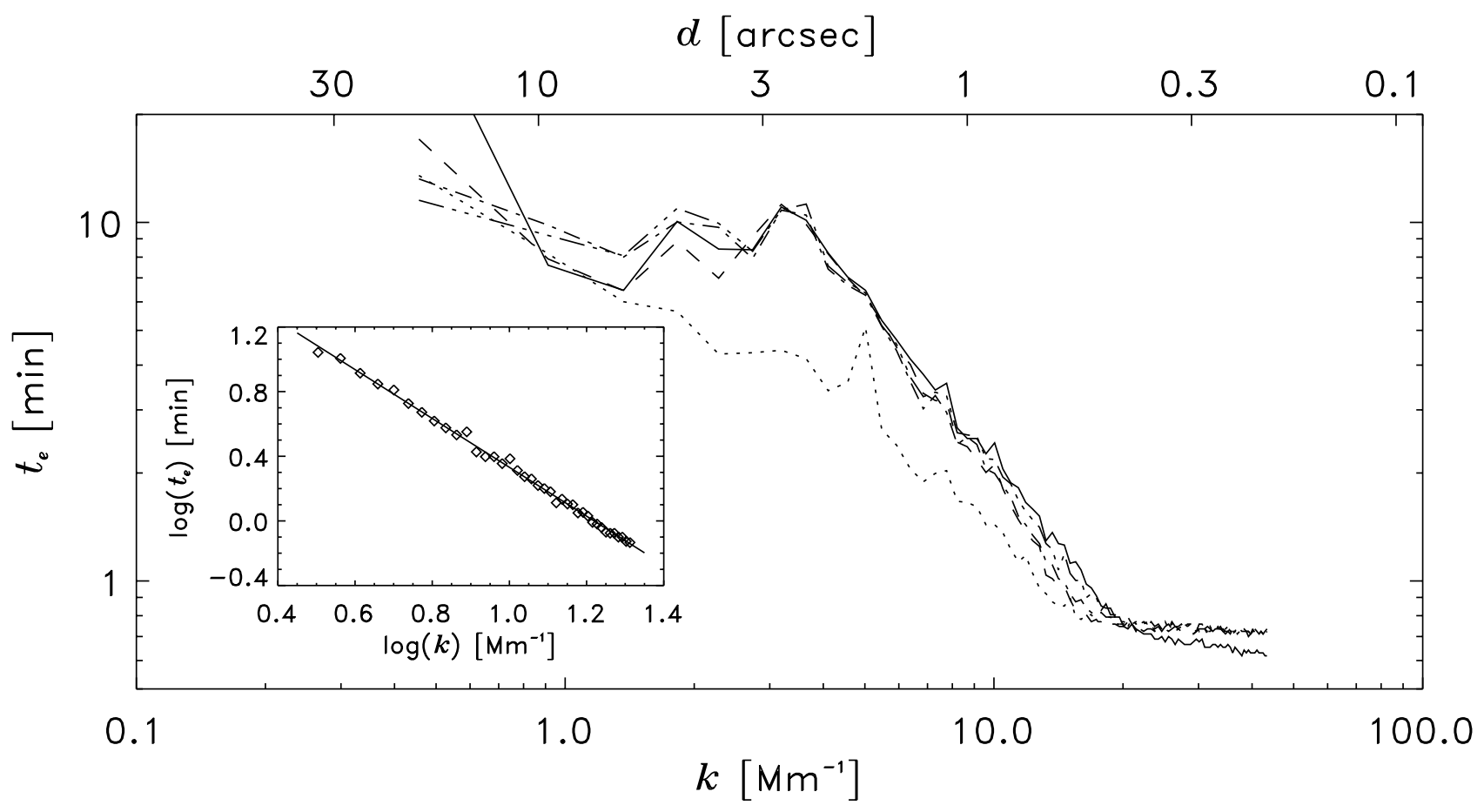

Fig. 12. Granular coherence $e$-folding times, $t_{\mathrm{e}}$, for broad band images (solid line), line core intensity maps (dotted line), intensity maps corresponding to $i=50 \%$ (dashed line), velocity maps from $i=0 \%$ (dash-dotted line) and from $i=50 \%$ (dash-dot-dot-dotted line). The inset shows the $e$-folding times for the broad band images in the range $3.2 \mathrm{Mm}^{-1}<k<20.5 \mathrm{Mm}^{-1}$ overplotted with a linear fit to them.

The temporal coherence analysis shows that the dependence of the lifetime of granules on structural size obeys a power law with an exponent of $\beta \approx 3 / 2$ with high significance. Many attempts for estimating granular lifetimes can be found in the literature (see e.g. Mehltretter 1978; Kawaguchi 1980; Dialetis et al. 1986; Title et al. 1989; Müller et al. 2001). The published mean values are in the range from $t=5 \mathrm{~min}$ up to $t=16 \mathrm{~min}$ although the lifetimes of individual features spread between $t<1 \mathrm{~min}$ (see e.g. Hirzberger et al. 1999) for the smallest structures up to $t>40 \mathrm{~min}$ (cf. e.g. Müller et al. 2001). The $e$-folding times estimated from the present data fit into these values although the maximum of $t_{\mathrm{e}}=11 \mathrm{~min}$ lies in the lower range of the published values. However, lifetimes estimated using cross-correlation methods are generally lower than those estimated from a tracking of individual granules (cf. Muller 1999; Alissandrakis et al. 1987).

Hirzberger et al. (1999) have measured granular lifetimes by tracking individual granules and have found a linear relation between granular areas and lifetimes (for granules with $r_{\mathrm{g}}<0$ 0'84). Kawaguchi (1980) has found a linear relation between size and lifetimes only for granules which end their "lives" dissolving into the background. The discrepancy with the present results might come from the effect that the coherence drops to very low values when the size of granules is significantly changing in time whereas a tracking algorithm - at least the one applied by Hirzberger et al. (1999) - is robust against temporal variations of granular sizes.

If one assumes that solar granulation is a manifestation of turbulent gas motions in the solar atmosphere the lifetimes of granules should obey the Kolmogorov-Oboukhov theory for isotropic turbulence (Kolmogorov 1941; Oboukhov 1941).
According to this theory a characteristic local time scale, $\tau(k)$, of the energy cascade can be written as $\tau(k)=\left[k^{3} E(k)\right]^{-1 / 2}$ where $E(k)$ denotes the turbulent energy spectrum depending on the wavenumber $k$. If it is assumed that $E(k) \sim k^{-\alpha}$ it follows that

$\tau(k) \sim k^{(\alpha-3) / 2} \quad$ or $\quad \beta=(3-\alpha) / 2$.

Comparing this power law with the result from Fig. 12, i.e. that $\beta=3 / 2$, leads to a scaling law for the turbulent energy spectrum with $\alpha=0$ which is incompatible with any theoretical scaling law for isotropic turbulence (e.g. with the Kolmogorov $\alpha=5 / 3$ law or with the $\alpha=17 / 3$ law for temperature fluctuations in an inertial conductive regime) and also with typical power spectra of solar granulation (see e.g. Fig. 9 or Espagnet et al. 1993). Hence, it has to be concluded that the KolmogorovOboukhov theory is not applicable to solar granulation. This is in agreement with numerical models of solar granulation of e.g. Steffen et al. (1989), Stein \& Nordlund (1998), or Gadun et al. (2000) which show much less turbulence as expected from the high Reynolds numbers in the solar photosphere.

Another possibility solving the contradictions between the different scaling laws might be that solar granulation is not an ergodic regime. The ergodic theorem is not yet proven for the Navier-Stokes equations (see Lesieur 1997). Therefore, the scaling laws of power spectra (spatial statistics) and that of the coherence spectra (temporal statistics) might be intrinsically incommensurable.

Acknowledgements. The author is grateful to Prof. F. Kneer and to an anonymous referee for fruitful comments on the manuscript. The Vacuum Tower Telescope is operated by the Kiepenheuer-Institut für 
Sonnenphysik in Freiburg (Germany) in the Spanish Observatorio del Teide of the Instituto de Astrofísica de Canarias in Tenerife. Financial support by the Austrian Fonds zur Förderung der wissenschaftlichen Forschung (Erwin-Schrödinger-Rückkehrprogramm No. R11) is gratefully acknowledged. The author thanks the Universitäts-Sternwarte Göttingen for the hospitality and the financial support.

\section{References}

Alissandrakis, C. E., Dialetis, D., \& Tsiropoula, G. 1987, A\&A, 174, 275

Berrilli, F., Consolini, G., Pietropaolo, E., et al. 2002, A\&A, 381, 253

Bovelet, B., \& Wiehr, E. 2001, Sol. Phys., 201, 13

Bray, R. J., \& Loughhead, R. E. 1984, A\&A, 133, 409

Carlier, A., Chauveau, F., Hugon, M., \& Rösch, J. 1968, Comp. Rend. Acad. Sci., 266, 119

de Boer, C. R. 1993, Ph.D. Thesis, Universität Göttingen

Deubner, F.-L. 1988, A\&A, 204, 301

Dialetis, D., Macris, C., Prokakis, D., \& Sarris, E. 1986, A\&A, 168, 330

Espagnet, O., Muller, R., Roudier, Th., \& Mein, N. 1993, A\&A, 271, 589

Espagnet, O., Muller, R., Roudier, Th., Mein, N., \& Mein, P. 1995, A\&AS, 109, 79

Gadun, A. S., Hanslmeier, A., Pikalov, K. N., et al. 2000, A\&AS, 146, 267

Hirzberger, J., Vázquez, M., Bonet, J. A., Hanslmeier, A., \& Sobotka, M. 1997, 1997, ApJ, 480, 406

Hirzberger, J., Bonet, J. A., Vázquez, M., \& Hanslmeier, A. 1999, ApJ, 515, 441

Hirzberger, J., Koschinsky, M., Kneer, F., \& Ritter, C. 2001, A\&A, 367,1011

Kawaguchi, I. 1980, Sol. Phys., 65, 207

Keil, S. L., \& Canfield, R. C. 1978, A\&A, 70169

Kolmogorov, A. N. 1941, Dokl. Akad. Nauk. SSSR, 30, 301

Komm, R., Mattig, W., \& Nesis, A. 1990, A\&A, 239, 340

Komm, R., Mattig, W., \& Nesis, A. 1991, A\&A, 252, 812

Krieg, J., Wunnenberg, M., Kneer, F., Koschinsky, M., \& Ritter, C. 1999, A\&A, 343, 983
Krieg, J., Kneer, F., Koschinsky, M., \& Ritter, C. 2000, A\&A, 360, 1157

Lesieur, M. 1997, Turbulence in Fluids (Kluwer, Dordrecht)

Mehltretter, J. P. 1978, A\&A, 62, 311

Müller, D. A. N., Steiner, O., Schlichenmaier, R., \& Brandt, P. N. 2001, Sol. Phys., 203, 211

Muller, R. 1999, The Solar Granulation, in Motions in the Solar Atmosphere, ed. A. Hanslmeier, \& M. Messerotti (Kluwer, Dordrecht), 35

Nesis, A., Durrant, C. J., \& Mattig, W. 1988, A\&A, 201, 153

Nesis, A., Hanslmeier, A., Hammer, R., et al. 1992, A\&A, 253, 561

Nesis, A., Hanslmeier, A., Hammer, R., et al. 1993, A\&A, 279, 599

Nesis, A., Hammer, R., Kiefer, M., et al. 1999, A\&A, 345, 265

Nordlund, Å., Spruit, H. C., Ludwig, H.-G., \& Trampedach, R. 1997, A\&A, 328, 229

Oboukhov, A. M. 1941, Dokl. Akad. Sci. Nauk. SSSR, 32A, 22

Oda, N. 1984, Sol. Phys., 93, 243

Rast, M. P. 1995, ApJ, 443, 863

Rast, M. P. 1999a, ApJ, 524, 462

Rast, M. P. 1999b, in High resolution solar physics: theory, observations, and techniques, ed. T. R. Rimmele, K. S. Balasubramaniam, \& R. R. Radick, ASP Conf. Ser., 183, 443

Rieutord, M., Roudier, Th., Malherbe, J. M., \& Rincon, F. 2000, A\&A, 357, 1063

Rimmele, T. R., Goode, P. R., Harold, E., \& Stebbins, R. T. 1995, ApJ, 444, L119

Roudier, Th., \& Muller, R. 1987, Sol. Phys., 107, 11

Roudier, Th., Eibe, M. T., Malherbe, J. M., et al. 2001, A\&A, 368, 652

Salucci, G., Bertello, L., Cavallini, F., Ceppatelli, G., \& Righini, A. 1994, A\&A, 285, 322

Simon, G. W., \& Leighton, R. B. 1964, ApJ, 140, 1120

Spruit, H. C., Nordlund, A., \& Title, A. M. 1990, ARA\&A, 28, 263

Steffen, M., Ludwig, H.-G., \& Krüß, A. 1989, A\&A, 213, 371

Stein, R. F., \& Nordlund, ̊. 1989, ApJ, 342, L95

Stein, R. F., \& Nordlund, Å. 1998, ApJ, 499, 914

Stein, R. F., \& Nordlund, Å. 2001, ApJ, 546, 585

Stoyan, D., \& Stoyan, H. 1992, Fraktale, Formen, Punktfelder (Akademie Verlag, Berlin)

Strous, L. H., Goode, P. R., \& Rimmele, T. R. 2000, ApJ, 535, 1000

Title, A. M., Tarbell, T. D., Topka, K. P., Ferguson, S. H., \& Shine, R. A., the SOUP Team, 1989, ApJ, 336, 475 